\title{
Integration via Modification: A Method of Reduction of Order for Systems of Ordinary Differential Equations
}

\author{
By \\ Pilar Ruiz Gordoa, ${ }^{*}$ Andrew Pickering ${ }^{* *}$ and Julia PradA ${ }^{* * *}$
}

\begin{abstract}
We give a method of reduction of order for systems of ordinary differential equations having a certain structure. Our method is based on establishing a Bäcklund transformation between an integrated modified system and an integrated version of the system under consideration. The originality of our approach lies in the application of ideas more familiar within the context of completely integrable partial differential equations to the question of reduction of order of ordinary differential equations, whether these last be integrable or not.
\end{abstract}

\section{$\S 1$. Introduction}

Given a system of ordinary differential equations (ODEs), it may not always be "integrable" in any of the well-defined meanings of that word. Thus, for example, it may not have a sufficient number of first integrals — with "sufficient" and type of integral to be specified — or it may not have an underlying

Communicated by T. Kawai. Received April 26, 2004. Revised September 13, 2004.

2000 Mathematics Subject Classification(s): 34A05, 35Q51, 35Q53, 37K05, 37K35.

Key words: ordinary differential equations, first integrals, Bäcklund transformations, modified systems

* Area de Matemática Aplicada, ESCET, Universidad Rey Juan Carlos, C/ Tulipán s/n, 28933 Móstoles, Madrid, Spain.

e-mail: pilar.gordoa@urjc.es

** Departamento de Matemáticas, Universidad de Salamanca, Plaza de la Merced 1, 37008 Salamanca, Spain. Address from 1 October 2004: Area de Matemática Aplicada, ESCET, Universidad Rey Juan Carlos, C/ Tulipán s/n, 28933 Móstoles, Madrid, Spain.

e-mail: andrew.pickering@urjc.es

*** Departamento de Matemáticas, Universidad de Salamanca, Plaza de la Merced 1, 37008 Salamanca, Spain.

e-mail: prada@usal.es

(C) 2006 Research Institute for Mathematical Sciences, Kyoto University. All rights reserved. 
linear problem (this last referring to the definition of "integrability" adopted in the present paper). In such a case, perhaps the best that can be hoped for is at least to be able to reduce the order of the system. This then becomes a question of using properties of the structure of the system. For example, as is well known, we can always reduce by one the order of any autonomous system. The aim of the present paper is to show that for systems having a certain structure, a reduction of order, sometimes quite drastic, is always possible, and this whether the system is integrable or not.

In fact, this paper arises from recent work on integrable ODEs $[1,2,3,4]$, these being derived as higher order analogues of the famous six Painlevé equations $[5,6,7,8]$. In this work we made use of the connection between nonisospectral scattering problems and linear problems for ODEs [9], and thus naturally were led to ODEs having a very similar structure to the partial differential equations (PDEs) to which they are related. This approach to obtaining higher order Painlevé equations is more general than that of simply taking similarity reductions of the higher order flows of a hierarchy, see e.g. [10], and provides an alternative to classical Painlevé classification, e.g. as undertaken in $[11,12,13,14,15]$. However, having obtained an ODE together with its underlying linear problem, there then remains the question of whether that ODE is of the minimum order possible. Does it admit first integrals? As a simple example, let us consider the similarity reduction of the Korteweg-de Vries (KdV) equation $[10,16]$,

$$
v_{t}+\left(v_{z z}+3 v^{2}\right)_{z}=0
$$

setting

$$
v(z, t)=\frac{u(x)}{(3 t)^{\frac{2}{3}}}, \quad x=\frac{z}{(3 t)^{\frac{1}{3}}},
$$

yields

$$
\left(u_{x x}+3 u^{2}\right)_{x}-x u_{x}-2 u=0,
$$

which integrates once [16] to give, with constant of integration $C$,

$$
(2 u-x) u_{x x}+(2 u-x)^{2} u-\left(u_{x}\right)^{2}+u_{x}=C .
$$

Thus, whilst it is of course correct that the ODEs (3) and (4) define the same function, this function is better described as being defined by a second order equation. In particular, given that (4) is related to the second Painlevé equation $\left(P_{I I}\right)$

$$
U_{x x}=2 U^{3}+x U+\alpha
$$


by a Bäcklund transformation (BT) $[8,10,16]$,

$$
u=U_{x}-U^{2}, \quad U=-\frac{u_{x}-\alpha}{2 u-x}, \quad C=\alpha(1-\alpha),
$$

this function is transcendental, but we would not refer to it as being a transcendental function obtained at third order. For this reason it is important to know if any reduction of order of an ODE believed to define a new transcendental function is possible. This is true independently of which of the methods in [1]-[15] are used to obtain the ODE. We show in fact that the integration of the ODE (3), properly understood, is a consequence of the BT (6) between equations (4) and (5).

The integration process presented here was discussed in [17] within the context of a particular example, namely, a fourth Painlevé hierarchy derived in [4] as an integrable hierarchy of ODEs related to the dispersive water wave hierarchy of [18]-[25]. In particular, it allowed a reduction of order by two for each member of this hierarchy of ODEs. Here we give a general formulation of this approach. Further, once phrased generally, we observe that our method is in fact applicable to a wide class of ODEs, both integrable and non-integrable. Since our approach is based on the factorization of Hamiltonian operators for PDEs under Miura maps [26], the technique presented here then represents a curious juxtaposition of ideas, in the sense that it represents the application of properties characteristic of integrable PDEs to the question of the reduction of order of integrable and non-integrable ODEs.

\section{$\S 2 . \quad$ Reduction of Order: a Simple Example}

Let us begin by explaining our approach within the context of equation (3). This equation can be written

$$
B[u](u-x / 2)=0,
$$

where

$$
B[u]=\partial_{x}^{3}+4 u \partial_{x}+2 u_{x}
$$

is one of the Hamiltonian operators of the KdV hierarchy [27] $\left(\partial_{x}=\partial / \partial x=\right.$ $d / d x$ for an ODE). As is well known, this operator factorizes under the Miura map

$$
u=F[U]=U_{x}-U^{2}
$$


to give

$$
\left.B[u]\right|_{u=F[U]}=F^{\prime}[U] \tilde{B}\left(F^{\prime}[U]\right)^{\dagger},
$$

where $\tilde{B}=-\partial_{x}$ is the Hamiltonian operator of the modified $\mathrm{KdV}(\mathrm{mKdV})$ hierarchy, $F^{\prime}[U]$ is the Fréchet derivative of $F[U]$, and $\left(F^{\prime}[U]\right)^{\dagger}$ is the adjoint of this last. It is the factorization (10) that forms the basis of the definition of a Miura map [26].

Considering this modification process, but now in the ODE case, then yields

$$
\tilde{B}\left(F^{\prime}[U]\right)^{\dagger}\left(U_{x}-U^{2}-x / 2\right)=0
$$

as a modified version of equation (7). That is, equation (11) has the same relation to equation (7) as the $\mathrm{mKdV}$ equation has to the $\mathrm{KdV}$ equation. Equation (11) is

$$
\partial_{x}\left(\partial_{x}+2 U\right)\left(U_{x}-U^{2}-x / 2\right)=0,
$$

which then gives immediately the integrated modified version of equation (7) as

$$
\left(\partial_{x}+2 U\right)\left(U_{x}-U^{2}-x / 2\right)=\alpha-1 / 2 \quad \text { or } \quad U_{x x}=2 U^{3}+x U+\alpha,
$$

where $\alpha$ is an arbitrary constant. Thus we see that we have effected a reduction of order, but for our modified equation. The question is then how to relate this integrated modified equation, which is in fact $P_{I I}(5)$, to our original equation (7).

The answer to this question lies in constructing a BT between our integrated modified equation and an integrated version of (7). In (13) we replace $U_{x}-U^{2}$ by $u$ to obtain, along with (9), the BT

$$
\begin{array}{r}
U(2 u-x)+\left(u_{x}-\alpha\right)=0, \\
u-U_{x}+U^{2}=0 .
\end{array}
$$

Solving (15) for $u$ and substituting into (14) yields (13). On the other hand, solving (14) for $U$ and substituting in (15) yields

$$
(2 u-x) u_{x x}+(2 u-x)^{2} u-\left(u_{x}\right)^{2}+u_{x}=\alpha(1-\alpha),
$$

and thus we recover the BT (14), (15) between $P_{I I}$ and (16). This BT is precisely that given in the Introduction, with (16) being the integrated version of (7). 
Thus we see that a first integral of equation (7) can be obtained by first passing to a modified equation, integrating that modified equation, and then returning to our original variables through the construction of a BT, this BT providing a mapping between the integrated modified equation and the integrated version of the original equation (7). Here the advantage is that the modified equation is much easier to integrate than the original equation. We note that although the integrated version of (7) is well known, as is the BT between this and $P_{I I}$, the integration procedure explained here, i.e. via modification, has not been given before. It is this integration procedure that interests us here.

\section{$\S 3 . \quad$ Reduction of Order via Modification}

We work as usual in the algebra of differential functions, i.e. of smooth functions of $x$, the variables $u_{i}(x), i=0, \ldots, N-1$, and their derivatives $u_{i, j x}=$ $\partial_{x}^{j} u_{i}(x)$ up to some finite but unspecified order $n$. We consider differential operators with coefficients matrices with entries in this algebra, which act on $N$-vectors, again with entries in this algebra. These we denote as $B[\mathbf{u}]$ and $\mathbf{L}[\mathbf{u}]$, respectively; we will also use the notation $\mathbf{u}=\left(u_{0}, u_{1}, \ldots, u_{N-1}\right)^{T}$. Further, we have defined as usual the notions of Hamiltonian operator and Miura map. For details we refer to $[27,26,28]$. We recall that in our ODE case $\partial_{x}$ is the total derivative operator $d / d x$.

We now formulate the ideas in the previous section as the following:

Theorem. Consider equations of the form $B[\mathbf{u}] \mathbf{L}[\mathbf{u}]=0$, where $B$ is a Hamiltonian operator. Let $\mathbf{u}=\mathbf{F}[\mathbf{U}]$, where $\mathbf{U}=\left(U_{0}, \ldots, U_{N-1}\right)^{T}$, be a Miura transformation such that:

(i) $\left.B[\mathbf{u}]\right|_{\mathbf{u}=\mathbf{F}[\mathbf{U}]}=\mathbf{F}^{\prime}[\mathbf{U}] \tilde{B}\left(\mathbf{F}^{\prime}[\mathbf{U}]\right)^{\dagger}$, where $\mathbf{F}^{\prime}[\mathbf{U}]$ is the Fréchet derivative of $\mathbf{F}[\mathbf{U}],\left(\mathbf{F}^{\prime}[\mathbf{U})^{\dagger}\right.$ its adjoint and $\tilde{B}=A \partial_{x}$ for some non-singular constant matrix A;

(ii) $\left(\mathbf{F}^{\prime}[\mathbf{U}]\right)^{\dagger} \mathbf{L}[\mathbf{u}]+\boldsymbol{\gamma}=0$, with $\boldsymbol{\gamma}=\left(\gamma_{0}, \ldots, \gamma_{N-1}\right)^{T}$ constant, is a linear system in the modified variables $U_{0}, \ldots, U_{N-1}$, for which it has the unique solution $\mathbf{U}=\mathbf{G}[\mathbf{u}, \gamma]$.

Then the pair of relations

$$
\begin{aligned}
& \left(\mathbf{F}^{\prime}[\mathbf{U}]\right)^{\dagger} \mathbf{L}[\mathbf{u}]+\gamma=0, \\
& \mathbf{u}=\mathbf{F}[\mathbf{U}]
\end{aligned}
$$


defines a Bäcklund transformation between the two systems

$$
\begin{aligned}
& \left(\mathbf{F}^{\prime}[\mathbf{U}]\right)^{\dagger} \mathbf{L}[\mathbf{F}[\mathbf{U}]]+\boldsymbol{\gamma}=0, \\
& \mathbf{u}-\mathbf{F}[\mathbf{G}[\mathbf{u}, \boldsymbol{\gamma}]]=0 .
\end{aligned}
$$

Moreover the second of these, $\mathbf{u}-\mathbf{F}[\mathbf{G}[\mathbf{u}, \boldsymbol{\gamma}]]=0$, represents an integrated version of the original system $B[\mathbf{u}] \mathbf{L}[\mathbf{u}]=0$.

Proof. Comparing the orders of $B[\mathbf{u}] \mathbf{L}[\mathbf{u}]=0$ and (20), which has $N$ integration constants, we see that we only need to show that if (20) holds, then so does the equation $B[\mathbf{u}] \mathbf{L}[\mathbf{u}]=0$ :

$$
\begin{aligned}
B[\mathbf{u}] L[\mathbf{u}] & =B[\mathbf{F}[\mathbf{G}[\mathbf{u}, \boldsymbol{\gamma}]]] \mathbf{L}[\mathbf{u}] \quad \text { by virtue of }(20) \\
& =\mathbf{F}^{\prime}[\mathbf{G}[\mathbf{u}, \boldsymbol{\gamma}]] \tilde{B}\left(\mathbf{F}^{\prime}[\mathbf{G}[\mathbf{u}, \boldsymbol{\gamma}]]\right)^{\dagger} \mathbf{L}[\mathbf{u}] \quad \text { using (i) } \\
& =\mathbf{F}^{\prime}[\mathbf{G}[\mathbf{u}, \boldsymbol{\gamma}]] \tilde{B}(-\boldsymbol{\gamma}) \quad \text { using (ii) } \\
& =0 \quad \text { since } \tilde{B}=A \partial_{x} .
\end{aligned}
$$

We see that the above Theorem retains the structure of the example discussed in Section 2, i.e. we consider passing to a modified equation $\tilde{B}\left(\mathbf{F}^{\prime}[\mathbf{U}]\right)^{\dagger} \mathbf{L}\left[\mathbf{F}[\mathbf{U}]=0\right.$ which, since $\tilde{B}=A \partial_{x}$, is easy to integrate; this integrated modified equation is then related to our original equation by means of a BT. We now make two remarks:

Remark One. It may sometimes be necessary to make a change of variables in order to put the system under consideration in the form $B[\mathbf{u}] \mathbf{L}[\mathbf{u}]=0$; see Example 4.1.

Remark Two. That the above Theorem is also applicable to non-integrable equations is a result of the observation that we have not made any requirements on the form of $\mathbf{L}[\mathbf{u}]$. It is thus indeed the case that we are using transformation properties of Hamiltonian operators under Miura maps — characteristic of integrable PDEs - in order to effect a reduction of order of systems of ODEs, integrable or not.

\section{§4. Examples}

\section{§4.1. A seventh order scalar example}

We consider as our first example the seventh order ODE

$$
\begin{array}{r}
{\left[u_{6 x}+14 u u_{4 x}+28 u_{x} u_{x x x}+21\left(u_{x x}\right)^{2}+70 u^{2} u_{x x}+70 u\left(u_{x}\right)^{2}+35 u^{4}\right]_{x}} \\
+g\left(4 u+2 x u_{x}\right)+4 h=0,
\end{array}
$$


where $g$ and $h$ are arbitrary constants. This is a particular member of the ODE hierarchy $\mathcal{R}^{n} u_{x}+g_{n-1}\left(4 u+2 x u_{x}\right)+g_{n}=0$, where $\mathcal{R}$ is the recursion operator of the KdV hierarchy (see equation (3.21) in [2]). We consider here the case $g \neq 0$, since the case $g=0$ is easily integrated. Without loss of generality we take $g=1$.

We note that this ODE is not of the form $B[u] L[u]=0$ for some $B[u]$ and $L[u]$. However, if we make the change of variable $u=v-h$, we obtain

$$
\begin{array}{r}
{\left[v_{6 x}+14 v v_{4 x}+28 v_{x} v_{x x x}+21\left(v_{x x}\right)^{2}+\right.} \\
\left.-14 h\left[v_{4 x}+10 v v_{x x}+5\left(v_{x}\right)^{2}+10 v^{3}\right]_{x}+70 v\left(v_{x}\right)^{2}+35 v^{4}\right]_{x}\left[v_{x x}+3 v^{2}\right]_{x} \\
-140 h^{3} v_{x}+\left(4 v+2 x v_{x}\right)=0 .
\end{array}
$$

This last is now in the required form $B[v] L[v]=0$ with

$$
\begin{aligned}
B[v]= & \partial_{x}^{3}+4 v \partial_{x}+2 v_{x} \\
L[v]= & v_{4 x}+10 v v_{x x}+5\left(v_{x}\right)^{2}+10 v^{3}-14 h\left[v_{x x}+3 v^{2}\right] \\
& +70 h^{2} v-70 h^{3}+x .
\end{aligned}
$$

Using the Miura map $v=F[U]=U_{x}-U^{2}$, as in Section 2, our Theorem gives that

$$
v=F[G[v, \gamma]]
$$

is an integrated version of equation (23), where $U=G[v, \gamma]$ is the solution of

$$
\left(F^{\prime}[U]\right)^{\dagger} L[v]+\gamma=0, \quad \text { i.e. of } \quad\left(\partial_{x}+2 U\right) L[v]-\gamma=0 .
$$

This then gives

$$
U=G[v, \gamma]=\frac{\gamma-\partial_{x} L[v]}{2 L[v]},
$$

and substituting in (26) leads to

$$
L[v]\left(\partial_{x}^{2} L[v]\right)-\frac{1}{2}\left(\partial_{x} L[v]\right)^{2}+2 v L[v]^{2}=-\frac{1}{2}\left(\gamma^{2}\right) .
$$

Finally, setting $v=u+h$, we obtain the first integral of (22), with $g=1$, as

$$
M[u]\left(\partial_{x}^{2} M[u]\right)-\frac{1}{2}\left(\partial_{x} M[u]\right)^{2}+2(u+h) M[u]^{2}=-\frac{1}{2}\left(\gamma^{2}\right),
$$


where

$M[u]=L[u+h]=u_{4 x}+10 u u_{x x}+5\left(u_{x}\right)^{2}+10 u^{3}-4 h\left[u_{x x}+3 u^{2}\right]+16 h^{2} u-32 h^{3}+x$.

Thus, even though our original equation was not in the form $B[u] L[u]=0$, we are still able to effect a reduction of order by one. We also note that explicit construction of the modified equation, or its integrated form, is not necessary. In fact, in this case, the integrated modified equation is equivalent to a special case of the sixth order member of the generalized second Painlevé hierarchy given in [29].

It might be pointed out that having put our system in the form $B[v] L[v]=$ 0 , integration to (29) is straightforward, since we have $L[u]$ as an integrating factor. However, the aim of the present paper is to avoid such ad-hoc considerations. We now turn to systems where spotting how to reduce their order is more difficult.

\section{§4.2. Multicomponent systems of ODEs}

Our examples in this section are based on the Hamiltonian structures and Miura maps of evolution equations of generalized KdV type associated to energy-dependent Schrödinger operators $[23,30]$. We thus consider $N$ component systems in the dependent variables $\mathbf{u}=\left(u_{0}, u_{1}, \ldots, u_{N-1}\right)^{T}$. We find that we are always able to reduce the order of the system by $N$. We first give our results in a general form, and then consider particular examples.

Consider systems of equations of, or which can be put into, the form

$$
B[\mathbf{u}] \mathbf{L}[\mathbf{u}]=0,
$$

where $B[\mathbf{u}]$ is the Hamiltonian operator

$$
B[\mathbf{u}]=\left(\begin{array}{ccccc} 
& & & J_{0} \\
& & & . & J_{1} \\
& & \cdot & . & \vdots \\
& \cdot & & \vdots \\
J_{0} & J_{1} & \ldots & J_{N-1}
\end{array}\right)
$$

with each

$$
J_{i}=\frac{1}{4} \varepsilon_{i} \partial_{x}^{3}+u_{i} \partial_{x}+\frac{1}{2} u_{i, x}
$$


where all $\varepsilon_{i}$ are constant. Then we have the Miura map $\mathbf{u}=\mathbf{F}[\mathbf{U}]$, where $\mathbf{U}=$ $\left(U_{0}, U_{1}, \ldots, U_{N-1}\right)^{T}$, given by

$$
u_{k}=-\sum_{i=0}^{k}\left(\alpha_{k-i} U_{i, x}+U_{i} U_{k-i}\right)
$$

with (and provided these relations are consistent)

$$
\varepsilon_{k}=\sum_{i=0}^{k} \alpha_{i} \alpha_{k-i}
$$

Under this Miura map the above Hamiltonian operator admits the factorization

$$
\left.B[\mathbf{u}]\right|_{\mathbf{u}=\mathbf{F}[\mathbf{U}]}=\mathbf{F}^{\prime}[\mathbf{U}] \tilde{B}\left(\mathbf{F}^{\prime}[\mathbf{U}]\right)^{\dagger},
$$

where $\mathbf{F}^{\prime}[\mathbf{U}]$ is the Fréchet derivative of the Miura map,

$$
\mathbf{F}^{\prime}[\mathbf{U}]=\left(\begin{array}{cccc}
f_{0} & & & \\
f_{1} & \ddots & & \\
\vdots & \ddots & \ddots & \\
f_{N-1} & \cdots & f_{1} & f_{0}
\end{array}\right),
$$

with each $f_{k}=-\alpha_{k} \partial_{x}-2 U_{k}$, and where $\tilde{B}$ is the Hamiltonian operator

$$
\tilde{B}=-\frac{1}{4}\left(\begin{array}{lll} 
& & \partial_{x} \\
& & \partial_{x} \\
\partial_{x} & &
\end{array}\right) .
$$

According to our Theorem,

$$
\mathbf{u}=\mathbf{F}[\mathbf{G}[\mathbf{u}, \boldsymbol{\gamma}]]
$$

is an integrated version of the system (32), where $\mathbf{U}=\mathbf{G}[\mathbf{u}, \boldsymbol{\gamma}]$ is the solution of

$$
\left(\mathbf{F}^{\prime}[\mathbf{U}]\right)^{\dagger} \mathbf{L}[\mathbf{u}]+\gamma=0 .
$$

Setting $\mathbf{L}[\mathbf{u}]=\left(L_{0}[\mathbf{u}], L_{1}[\mathbf{u}], \ldots, L_{N-1}[\mathbf{u}]\right)^{T}$, we find that this last equation reads 
(42)

$$
C\left(\begin{array}{c}
U_{0} \\
U_{1} \\
\vdots \\
U_{N-1}
\end{array}\right)=\frac{1}{2}\left(\begin{array}{c}
\alpha_{0} \partial_{x} L_{0}[\mathbf{u}]+\alpha_{1} \partial_{x} L_{1}[\mathbf{u}] \ldots+\alpha_{N-1} \partial_{x} L_{N-1}[\mathbf{u}]+\gamma_{0} \\
\alpha_{0} \partial_{x} L_{1}[\mathbf{u}] \ldots+\alpha_{N-2} \partial_{x} L_{N-1}[\mathbf{u}]+\gamma_{1} \\
\vdots \\
\alpha_{0} \partial_{x} L_{N-1}[\mathbf{u}]+\gamma_{N-1}
\end{array}\right)
$$

where

$$
C=\left(\begin{array}{cccc}
L_{0}[\mathbf{u}] & L_{1}[\mathbf{u}] & \ldots & L_{N-1}[\mathbf{u}] \\
L_{1}[\mathbf{u}] & \ldots & L_{N-1}[\mathbf{u}] \\
\vdots & \cdots & & \\
L_{N-1}[\mathbf{u}] & &
\end{array}\right)
$$

Thus provided $L_{N-1}[\mathbf{u}] \neq 0$, we can solve for the modified variables as

$$
\mathbf{U}=\mathbf{G}[\mathbf{u}, \gamma]=\frac{1}{2} C^{-1}\left(\begin{array}{c}
\alpha_{0} \partial_{x} L_{0}[\mathbf{u}]+\alpha_{1} \partial_{x} L_{1}[\mathbf{u}] \ldots+\alpha_{N-1} \partial_{x} L_{N-1}[\mathbf{u}]+\gamma_{0} \\
\alpha_{0} \partial_{x} L_{1}[\mathbf{u}] \ldots+\alpha_{N-2} \partial_{x} L_{N-1}[\mathbf{u}]+\gamma_{1} \\
\vdots \\
\alpha_{0} \partial_{x} L_{N-1}[\mathbf{u}]+\gamma_{N-1}
\end{array}\right),
$$

and substitution into (40) then gives the integrated version of equation (32). We note that the integrated modified equation results from the substitution of $\mathbf{u}=\mathbf{F}[\mathbf{U}]$ into (41), or equivalently into (42). Thus we see that, provided that $L_{N-1}[\mathbf{u}] \neq 0$, and provided that the relations (36) are consistent — for which a sufficient condition, for example, is that $\varepsilon_{0} \neq 0$ - we are always able to effect a reduction of the order of our system (32) by $N$. This is true whatever the form of $\mathbf{L}[\mathbf{u}]$.

\subsubsection{Particular examples}

As a particular example of such a multicomponent system we take

$$
\mathbf{L}[\mathbf{u}]=\left(0, \ldots, 0,2, u_{N-1}+g x\right)^{T}
$$

where $g$ is constant. In the case $N=2$ and $\varepsilon_{0} \neq 0=\varepsilon_{1}$, this corresponds to a nonautonomous extension of the stationary flow of the dispersive water wave system. We assume $L_{N-1}[\mathbf{u}] \neq 0$; the case $L_{N-1}[\mathbf{u}]=0$ leads only to the trivial solution $u_{N-1}=-g x$ and all $u_{i}=c_{i}, i=0, \ldots, N-2$, each $c_{i}$ an arbitrary constant.

In general, i.e. where at least one $\varepsilon_{i} \neq 0$, the resulting system $(32)$ is of order $N+2$. In the case where all $\varepsilon_{i}=0$, it is of order $N$. Thus our reduction of 
order by $N$ leads respectively either to a second order system, or, remarkably, to a complete integration of the system. Let us consider now the details of this reduction process.

With the choice (45), $C^{-1}$ reads

$$
C^{-1}=\left(\begin{array}{ccc} 
& & \frac{1}{L_{N-1}[\mathbf{u}]} \\
& \frac{1}{L_{N-1}[\mathbf{u}]} & -\frac{L_{N-2}[\mathbf{u}]}{\left(L_{N-1}[\mathbf{u}]\right)^{2}} \\
. \cdot & . \cdot & \vdots \\
\frac{1}{L_{N-1}[\mathbf{u}]}-\frac{L_{N-2}[\mathbf{u}]}{\left(L_{N-1}[\mathbf{u}]\right)^{2}} & \ldots & (-1)^{N-1} \frac{\left(L_{N-2}[\mathbf{u}]\right)^{N-1}}{\left(L_{N-1}[\mathbf{u}]\right)^{N}}
\end{array}\right),
$$

with $L_{N-2}[\mathbf{u}]=2$ and $L_{N-1}[\mathbf{u}]=u_{N-1}+g x$, and (44) then gives - where we use the fact that $\partial_{x} L_{N-2}[\mathbf{u}]=0-$

$$
U_{i}=\frac{1}{2} \sum_{l=0}^{i}(-1)^{l} \frac{\left(L_{N-2}[\mathbf{u}]\right)^{l}}{\left(L_{N-1}[\mathbf{u}]\right)^{l+1}}\left(\alpha_{i-l} \partial_{x} L_{N-1}[\mathbf{u}]+\gamma_{N-1-i+l}\right) .
$$

The integrated version of (32) is obtained by substituting these expressions for $U_{i}$ into the Miura map (35). The first $N-1$ equations of the Miura map define $u_{0}, u_{1}, \ldots, u_{N-2}$ in terms of $u_{N-1}$. The last equation of the Miura map,

$$
u_{N-1}=-\sum_{i=0}^{N-1}\left(\alpha_{N-1-i} U_{i, x}+U_{i} U_{N-1-i}\right),
$$

in the case where at least one $\varepsilon_{i} \neq 0$, then gives a second order ODE in $u_{N-1}$. In the case where all $\varepsilon_{i}=0$, where we also take all $\alpha_{j}=0$, this last equation of the Miura map allows recovery of $u_{N-1}$ as an algebraic function of $x$.

We now consider as explicit examples the two and three component cases:

- For $N=2$ we have the system of equations

$$
\begin{aligned}
& \frac{1}{4} \varepsilon_{0} u_{1, x x x}+u_{0} u_{1, x}+\frac{1}{2} u_{0, x} u_{1}+g\left(u_{0}+\frac{1}{2} x u_{0, x}\right)=0, \\
& u_{0, x}+\frac{1}{4} \varepsilon_{1} u_{1, x x x}+\frac{3}{2} u_{1} u_{1, x}+g\left(u_{1}+\frac{1}{2} x u_{1, x}\right)=0,
\end{aligned}
$$

which is either a fourth order system (when at least one $\varepsilon_{i} \neq 0$ ) or a second order system (when all $\varepsilon_{i}=0$ ). The Miura map (35) is

$$
\begin{aligned}
& u_{0}=-\alpha_{0} U_{0, x}-U_{0}^{2}, \\
& u_{1}=-\alpha_{1} U_{0, x}-\alpha_{0} U_{1, x}-2 U_{0} U_{1},
\end{aligned}
$$


and the equations corresponding to (47) are

$$
\begin{aligned}
U_{0} & =\frac{\alpha_{0} \partial_{x} L_{1}+\gamma_{1}}{2 L_{1}}, \\
U_{1} & =\frac{L_{1}\left(\alpha_{1} \partial_{x} L_{1}+\gamma_{0}\right)-L_{0}\left(\alpha_{0} \partial_{x} L_{1}+\gamma_{1}\right)}{2 L_{1}^{2}},
\end{aligned}
$$

where $\varepsilon_{0}=\alpha_{0}^{2}, \varepsilon_{1}=2 \alpha_{0} \alpha_{1}, L_{0}=2$ and $L_{1}=u_{1}+g x$. Substitution of these expressions for $U_{0}$ and $U_{1}$ into the Miura map yields the system of integrated equations:

$$
\begin{aligned}
u_{0}= & \frac{\varepsilon_{0}\left(u_{1, x}+g\right)^{2}-\gamma_{1}^{2}}{4\left(u_{1}+g x\right)^{2}}-\frac{\varepsilon_{0} u_{1, x x}}{2\left(u_{1}+g x\right)} \\
u_{1}= & \frac{-\varepsilon_{0}\left(u_{1, x}+g\right)^{2}+\gamma_{1}^{2}}{\left(u_{1}+g x\right)^{3}}+\frac{\varepsilon_{1}\left(u_{1, x}+g\right)^{2}+4 \varepsilon_{0} u_{1, x x}-2 \gamma_{0} \gamma_{1}}{4\left(u_{1}+g x\right)^{2}} \\
& -\frac{\varepsilon_{1} u_{1, x x}}{2\left(u_{1}+g x\right)} .
\end{aligned}
$$

The first of these then gives $u_{0}$ in terms of $u_{1}$. When at least one $\varepsilon_{i} \neq 0$, the second equation is a second order ODE for $u_{1}$, and we have effected a reduction of order by two; when all $\varepsilon_{i}=0$, this second equation defines $u_{1}$ as an algebraic function of $x$, and thus we have a complete integration of the system.

We note that our calculation of the above integrated system is valid provided that the relations $\varepsilon_{0}=\alpha_{0}^{2}, \varepsilon_{1}=2 \alpha_{0} \alpha_{1}$ are consistent. The only case in which these relations are not consistent is $\varepsilon_{0}=0 \neq \varepsilon_{1}$, which corresponds in the PDE case to Ito's equation [31], and for which the difficulties of obtaining a Miura map are well known [30, 32]; see also Section 4.3. However, we note that the above formulae still give an integrated version of the system (49), (50), even in this troublesome case.

- For $N=3$ we have the system of equations

$$
\begin{aligned}
& \frac{1}{4} \varepsilon_{0} u_{2, x x x}+u_{0} u_{2, x}+\frac{1}{2} u_{0, x} u_{2}+g\left(u_{0}+\frac{1}{2} x u_{0, x}\right)=0, \\
& u_{0, x}+\frac{1}{4} \varepsilon_{1} u_{2, x x x}+u_{1} u_{2, x}+\frac{1}{2} u_{1, x} u_{2}+g\left(u_{1}+\frac{1}{2} x u_{1, x}\right)=0, \\
& u_{1, x}+\frac{1}{4} \varepsilon_{2} u_{2, x x x}+\frac{3}{2} u_{2} u_{2, x}+g\left(u_{2}+\frac{1}{2} x u_{2, x}\right)=0,
\end{aligned}
$$


which is either of fifth order or third order. The Miura map is given by

$$
\begin{aligned}
& u_{0}=-\alpha_{0} U_{0, x}-U_{0}^{2}, \\
& u_{1}=-\alpha_{1} U_{0, x}-\alpha_{0} U_{1, x}-2 U_{0} U_{1}, \\
& u_{2}=-\alpha_{2} U_{0, x}-\alpha_{1} U_{1, x}-\alpha_{0} U_{2, x}-U_{1}^{2}-2 U_{0} U_{2},
\end{aligned}
$$

and the expressions for $U_{0}, U_{1}$ and $U_{2}$, corresponding to (47), are

$$
\begin{aligned}
& U_{0}=\frac{\alpha_{0} \partial_{x} L_{2}+\gamma_{2}}{2 L_{2}} \\
& U_{1}=\frac{L_{2}\left(\alpha_{1} \partial_{x} L_{2}+\gamma_{1}\right)-L_{1}\left(\alpha_{0} \partial_{x} L_{2}+\gamma_{2}\right)}{2 L_{2}^{2}} \\
& U_{2}=\frac{L_{2}^{2}\left(\alpha_{2} \partial_{x} L_{2}+\gamma_{0}\right)-L_{1} L_{2}\left(\alpha_{1} \partial_{x} L_{2}+\gamma_{1}\right)+L_{1}^{2}\left(\alpha_{0} \partial_{x} L_{2}+\gamma_{2}\right)}{2 L_{2}^{3}},
\end{aligned}
$$

where $\varepsilon_{0}=\alpha_{0}^{2}, \varepsilon_{1}=2 \alpha_{0} \alpha_{1}, \varepsilon_{2}=\alpha_{1}^{2}+2 \alpha_{0} \alpha_{2}, L_{0}=0, L_{1}=2$ and $L_{2}=u_{2}+g x$. Substituting into the Miura map then gives the integrated system

$$
\begin{aligned}
u_{0}= & \frac{\varepsilon_{0}\left(u_{2, x}+g\right)^{2}-\gamma_{2}^{2}}{4\left(u_{2}+g x\right)^{2}}-\frac{\varepsilon_{0} u_{2, x x}}{2\left(u_{2}+g x\right)}, \\
u_{1}= & \frac{-\varepsilon_{0}\left(u_{2, x}+g\right)^{2}+\gamma_{2}^{2}}{\left(u_{2}+g x\right)^{3}}+\frac{\varepsilon_{1}\left(u_{2, x}+g\right)^{2}+4 \varepsilon_{0} u_{2, x x}-2 \gamma_{1} \gamma_{2}}{4\left(u_{2}+g x\right)^{2}} \\
& -\frac{\varepsilon_{1} u_{2, x x}}{2\left(u_{2}+g x\right)}, \\
u_{2}= & 3 \frac{\varepsilon_{0}\left(u_{2, x}+g\right)^{2}-\gamma_{2}^{2}}{\left(u_{2}+g x\right)^{4}}-\frac{\varepsilon_{1}\left(u_{2, x}+g\right)^{2}+2 \varepsilon_{0} u_{2, x x}-2 \gamma_{1} \gamma_{2}}{\left(u_{2}+g x\right)^{3}} \\
& +\frac{\varepsilon_{2}\left(u_{2, x}+g\right)^{2}+4 \varepsilon_{1} u_{2, x x}-2 \gamma_{0} \gamma_{2}-\gamma_{1}^{2}}{4\left(u_{2}+g x\right)^{2}}-\frac{\varepsilon_{2} u_{2, x x}}{2\left(u_{2}+g x\right)},
\end{aligned}
$$

which is of order three less than (57)-(59). Thus, in the general case, we reduce a fifth order system to one of second order; in the special case where all $\varepsilon_{i}=0$, we effect a complete integration of our system.

We remark that the complete integration of our $N^{\text {th }}$ order systems when all $\varepsilon_{i}=0$ is presumably related to the invertibility of the operator $B[\mathbf{u}]$ in this case; our approach then corresponds to making an invertible change of variables (the Miura map) which greatly simplifies the process of obtaining the general solution. 


\section{§4.3. An example of Ito type}

We now consider two-component systems of, or which can be put into, the form

$$
B[\mathbf{u}] \mathbf{L}[\mathbf{u}]=0
$$

where $B[\mathbf{u}]$ is the Hamiltonian operator

$$
B=\left(\begin{array}{cc}
J_{1} & J_{0}-\partial \\
J_{0}-\partial & J_{1}
\end{array}\right),
$$

each $J_{i}$ being as defined in (34), with $\varepsilon_{0}=0 \neq \varepsilon_{1}$. It is the factorization of this Hamiltonian operator that allows the construction of modifications of Ito's equation [32]. This factorization reads

$$
\left.B[\mathbf{u}]\right|_{\mathbf{u}=\mathbf{F}[\mathbf{U}]}=\mathbf{F}^{\prime}[\mathbf{U}] \tilde{B}\left(\mathbf{F}^{\prime}[\mathbf{U}]\right)^{\dagger},
$$

where

$$
\tilde{B}=-\frac{1}{4}\left(\begin{array}{ll}
\partial & 0 \\
0 & \partial
\end{array}\right)
$$

and

$$
F^{\prime}=\left(\begin{array}{cc}
\alpha \partial-2 U_{1} & -2 U_{0} \\
-2 U_{0} & \alpha \partial-2 U_{1}
\end{array}\right)
$$

is the Fréchet derivative of the Miura map $\mathbf{u}=\mathbf{F}[\mathbf{U}]$ given by the pair of equations

$$
\begin{aligned}
& u_{0}=\alpha U_{0, x}-2 U_{0} U_{1}+1, \\
& u_{1}=\alpha U_{1, x}-U_{0}^{2}-U_{1}^{2},
\end{aligned}
$$

and where $\alpha^{2}=\varepsilon_{1}$.

According to our Theorem,

$$
\mathbf{u}=\mathbf{F}[\mathbf{G}[\mathbf{u}, \gamma]]
$$

is an integrated version of the system (69), where $\mathbf{U}=\mathbf{G}[\mathbf{u}, \gamma]$ is the solution of

$$
\left(\mathbf{F}^{\prime}[\mathbf{U}]\right)^{\dagger} \mathbf{L}[\mathbf{u}]+\gamma=0
$$


Setting $\mathbf{L}[\mathbf{u}]=\left(L_{0}[\mathbf{u}], L_{1}[\mathbf{u}]\right)^{T}$, we find that this last equation reads

$$
\left(\begin{array}{ll}
L_{1}[\mathbf{u}] & L_{0}[\mathbf{u}] \\
L_{0}[\mathbf{u}] & L_{1}[\mathbf{u}]
\end{array}\right)\left(\begin{array}{c}
U_{0} \\
U_{1}
\end{array}\right)=\frac{1}{2}\left(\begin{array}{l}
-\alpha \partial_{x} L_{0}[\mathbf{u}]+\gamma_{0} \\
-\alpha \partial_{x} L_{1}[\mathbf{u}]+\gamma_{1}
\end{array}\right)
$$

and so, provided that $\left(L_{1}[\mathbf{u}]\right)^{2}-\left(L_{0}[\mathbf{u}]\right)^{2} \neq 0$, we can solve for the modified variables as

$$
\mathbf{U}=\mathbf{G}[\mathbf{u}, \boldsymbol{\gamma}]=\frac{1}{2} \frac{1}{\left(L_{1}[\mathbf{u}]\right)^{2}-\left(L_{0}[\mathbf{u}]\right)^{2}}\left(\begin{array}{cc}
L_{1}[\mathbf{u}] & -L_{0}[\mathbf{u}] \\
-L_{0}[\mathbf{u}] & L_{1}[\mathbf{u}]
\end{array}\right)\left(\begin{array}{c}
-\alpha \partial_{x} L_{0}[\mathbf{u}]+\gamma_{0} \\
-\alpha \partial_{x} L_{1}[\mathbf{u}]+\gamma_{1}
\end{array}\right)
$$

and substitution into the Miura map then gives an integrated version of (69). The integrated modified system results from the substitution of $\mathbf{u}=\mathbf{F}[\mathbf{U}]$ into (77).

\subsubsection{A particular example}

We consider as a particular example the system of equations

$$
\begin{aligned}
& u_{0} u_{1, x}+\frac{1}{2} u_{0, x} u_{1}+g\left(u_{0}+\frac{1}{2} x u_{0, x}-1\right)=0, \\
& u_{0, x}+\frac{1}{4} \varepsilon_{1} u_{1, x x x}+\frac{3}{2} u_{1} u_{1, x}+g\left(u_{1}+\frac{1}{2} x u_{1, x}\right)=0,
\end{aligned}
$$

where $g$ is a constant. This system corresponds to the choice $\mathbf{L}[\mathbf{u}]=\left(2, u_{1}+\right.$ $g x)^{T}$, and is a nonautonomous extension of the stationary flow of Ito's equation.

We assume $\left(L_{1}[\mathbf{u}]\right)^{2}-\left(L_{0}[\mathbf{u}]\right)^{2} \neq 0$; the case $L_{1}[\mathbf{u}]= \pm L_{0}[\mathbf{u}]$ leads only to the trivial solution $u_{1}=-g x \pm 2, u_{0}= \pm g x+c, c$ an arbitrary constant.

Proceeding as in our previous examples, we solve for the modified variables,

$$
\begin{aligned}
& U_{0}=\frac{2 \alpha\left(u_{1, x}+g\right)+\gamma_{0}\left(u_{1}+g x\right)-2 \gamma_{1}}{2\left[\left(u_{1}+g x\right)^{2}-4\right]}, \\
& U_{1}=-\frac{\alpha\left(u_{1}+g x\right)\left(u_{1, x}+g\right)-\gamma_{1}\left(u_{1}+g x\right)+2 \gamma_{0}}{2\left[\left(u_{1}+g x\right)^{2}-4\right]},
\end{aligned}
$$

and substitute into the Miura map, which leads to 
(84)

$$
\begin{aligned}
u_{0}= & \frac{\varepsilon_{1} u_{1, x x}}{\left[\left(u_{1}+g x\right)^{2}-4\right]}-\frac{\varepsilon_{1}\left(u_{1}+g x\right) u_{1, x}^{2}}{\left[\left(u_{1}+g x\right)^{2}-4\right]^{2}}-\frac{2 g \varepsilon_{1}\left(u_{1}+g x\right) u_{1, x}}{\left[\left(u_{1}+g x\right)^{2}-4\right]^{2}} \\
& +\frac{u_{1}^{4}+4 g x u_{1}^{3}+\left(6 g^{2} x^{2}-\delta\right) u_{1}^{2}+\left(\beta+4 g^{3} x^{3}-2 g \delta x\right) u_{1}}{\left[\left(u_{1}+g x\right)^{2}-4\right]^{2}} \\
& +\frac{\left(g^{4} x^{4}-g^{2} \delta x^{2}+\beta g x-4 \delta+48\right)}{\left[\left(u_{1}+g x\right)^{2}-4\right]^{2}},
\end{aligned}
$$

$$
\begin{aligned}
0= & -2 \varepsilon_{1}\left(u_{1}+g x\right) u_{1, x x}+\frac{\varepsilon_{1}\left(u_{1}^{2}+g^{2} x^{2}+2 g x u_{1}+4\right)}{\left[\left(u_{1}+g x\right)^{2}-4\right]}\left(u_{1, x}^{2}+2 g u_{1, x}\right) \\
& -\frac{4 u_{1}^{5}+16 g x u_{1}^{4}+\left(24 g^{2} x^{2}-32\right) u_{1}^{3}+\left(16 g^{3} x^{3}-64 g x+\beta\right) u_{1}^{2}}{\left[\left(u_{1}+g x\right)^{2}-4\right]} \\
& +\frac{\left(32 g^{2} x^{2}-4 g^{4} x^{4}-2 g \beta x+16 \delta-192\right) u_{1}-g^{2} \beta x^{2}+16 g \delta x-128 g x-4 \beta}{\left[\left(u_{1}+g x\right)^{2}-4\right]}
\end{aligned}
$$

where we now take as constants of integration $\beta$ and $\delta$ defined via $\beta=\gamma_{0}^{2}+$ $\gamma_{1}^{2}-\varepsilon_{1} g^{2}$ and $\delta=\left(\gamma_{0} \gamma_{1} / 2\right)+8$. The system (84), (85) is an integrated version of the system (80), (81); the first equation defines $u_{0}$ in terms of $u_{1}$, and the second is a second order ODE for $u_{1}$. Thus we have reduced the order of our system from four to two.

\section{$\S 5 . \quad$ Conclusions}

We have given a method of reduction of order for systems of ODEs having a certain structure. For some systems, this approach even allows a complete integration. This method, based on ideas more familiar within the context of completely integrable PDEs, is applicable to both integrable and non-integrable systems.

One important possible application of our results is to integrable ODEs believed to define new transcendental functions, where it might be possible to effect a reduction of order by exploiting their links to completely integrable PDEs; see Example 4.1 and also [17]. However, it is also clear that our approach has applications to any ODE related in a suitable way to a completely integrable $\mathrm{PDE}$, and beyond this to any ODE that happens to have the form required in order to apply our Theorem.

We have also given a variety of illustrative examples. Here it is worth pointing out that what is important in these examples is the factorization of $B[\mathbf{u}]$ and not the particular choice of $\mathbf{L}[\mathbf{u}]$. Thus, whilst the order of the system depends also on $\mathbf{L}[\mathbf{u}]$ — in our particular examples in Sections 4.2.1 
and 4.3.1, we have, for reasons of clarity, taken only very simple forms of $\mathbf{L}[\mathbf{u}]$, not depending on derivatives of the $u_{i}$ — we can always, for virtually any choice of $\mathbf{L}[\mathbf{u}]$, effect an $N$-fold integration.

In later papers we will consider further extensions of our approach, for example to systems where the Hamiltonian operator of the modified system is not of the simple form $A \partial_{x}$, or to cases where only weaker versions of our results are possible. With regard to this last, we note that for some systems, an $N$-fold integration is not possible, but partial results can still be obtained whereby a $p$-fold integration, for some $p<N$, can be performed; that is, we only obtain $p<N$ first integrals.

\section{Acknowledgements}

The research in this paper was supported in part by the DGESYC under contracts BFM2002-02609 and BFM2003-00078, and by the Junta de Castilla y León under contract SA011/04. PRG currently holds a research fellowship awarded by the Ministry of Science and Technology of Spain under the Programa Ramón y Cajal, which support is gratefully acknowledged.

\section{References}

[1] Gordoa, P. R. and Pickering, A., Bäcklund transformations for two new integrable partial differential equations, Europhys. Lett., 47 (1999), 21-24.

[2] Non-isospectral scattering problems: a key to integrable hierarchies, J. Math. Phys., 40 (1999), 5749-5786.

[3] (2000), 557-567.

[4] Gordoa, P. R., Joshi, N. and Pickering, A., On a generalized $2+1$ dispersive water wave hierarchy, Publ. RIMS, Kyoto Univ., 37 (2001), 327-347.

[5] Painlevé, P., Mémoire sur les équations différentielles dont l'intégrale générale est uniforme, Bull. Soc. Math. France, 28 (1900), 201-261.

[6] - Sur les équations différentielles du second ordre et d'ordre supérieur dont l'intégrale générale est uniforme, Acta Math., 25 (1902), 1-85.

[7] Gambier, B., Sur les équations différentielles du second ordre et du premier degré dont l'intégrale générale est à points critiques fixes, Acta Math., 33 (1910), 1-55.

[8] Ince, E. L., Ordinary Differential Equations, Dover, New York, 1956.

[9] Levi, D., Ragnisco, O. and Rodriguez, M. A., On non-isospectral flows, Painlevé equations and symmetries of differential and difference equations, Teoret. Mat. Fiz., 93 (1992), 473-480; Theoret. and Math. Phys., 93 (1993), 1409-1414.

[10] Airault, H., Rational solutions of Painlevé equations, Stud. Appl. Math., 61 (1979), 31-53.

[11] Chazy, J., Sur les équations différentielles du troisième ordre et d'ordre supérieur dont l'intégrale générale a ses points critiques fixes, Acta Math., 34 (1911), 317-385.

[12] Bureau, F. J., Differential equations with fixed critical points, Ann. Mat. Pura Appl., 66 (1964), 1-116.

[13] Muğan, U. and Jrad, F., Painlevé test and the first Painlevé hierarchy J. Phys. A., 32 (1999), 7933-7952. 
[14] Muğan, U. and Jrad, F., Painlevé test and higher order differential equations J. Nonlinear Math. Phys., 9 (2002), 282-310.

[15] - Non-polynomial third order equations which pass the Painlevé test, Z. Naturforsch A, 59 (2004), 163-180.

[16] Fokas, A. S. and Ablowitz, M. J., On a unified approach to transformations and elementary solutions of Painlevé equations J. Math. Phys., 23 (1982), 2033-2042.

[17] Pickering, A., Painlevé hierarchies and the Painlevé test, Teoret. Mat. Fiz., 137 (2003), 445-456; Theoret. and Math. Phys., 137 (2003), 1733-1742.

[18] Broer, L. J. F., Approximate equations for long water waves, Appl. Sci. Res., 31 (1975), 377-395.

[19] Kaup, D. J., Finding eigenvalue problems for solving nonlinear evolution equations, Progr. Theoret. Phys., 54 (1975), 72-78.

[20] oret. Phys., 54 (1975), 396-408.

[21] Jaulent, M. and Miodek, J., Nonlinear evolution equations associated with energydependent Schrödinger potentials, Lett. Math. Phys., 1 (1976), 243-250.

[22] Matveev, V. B. and Yavor, M. I., Solutions presque périodiques et à $N$-solitons de l'équation hydrodynamique non linéaire de Kaup, Ann. Inst. H. Poincaré Sect. A (N.S.), 31 (1979), 25-41.

[23] Martínez Alonso, L., Schrödinger spectral problems with energy-dependent potentials as sources of nonlinear Hamiltonian evolution equations, J. Math. Phys., 21 (1980), 2342-2349.

[24] Kupershmidt, B. A., Mathematics of dispersive water waves, Comm. Math. Phys., 99 (1985), 51-73.

[25] Sachs, R. L., On the integrable variant of the Boussinesq system: Painlevé property, rational solutions, a related many-body system, and equivalence with the AKNS hierarchy, Phys. D, 30 (1988), 1-27.

[26] Kupershmidt, B. A. and Wilson, G., Modifying Lax equations and the second Hamiltonian structure, Invent. Math., 62 (1981), 403-436.

[27] Magri, F., A simple model of the integrable Hamiltonian equation, J. Math. Phys., 19 (1978), 1156-1162.

[28] Olver, P. J. Applications of Lie Groups to Differential Equations, Springer-Verlag, New York, 1986.

[29] Pickering, A. Coalescence limits for higher order Painlevé equations, Phys. Lett. A, 301 (2002), 275-280.

[30] Antonowicz, M. and Fordy, A. P., Factorisation of energy dependent Schrödinger operators: Miura maps and modified systems, Comm. Math. Phys., 124 (1989), 465-486.

[31] Ito, M., Symmetries and conservation laws of a coupled nonlinear wave equation, Phys. Lett. A, 91 (1982), 335-338.

[32] Liu, Q. P. and Marshall, I., Two modifications of Ito's equation, Phys. Lett. A, 160 (1991), 155-160. 